\title{
The influence of the steep medial posterior tibial slope on medial meniscus tears in adolescent patients: a retrospective case- control study
}

Xiangtian Deng ${ }^{1,2 \dagger}$, Hongzhi Hu ${ }^{3 \dagger}$, Qingcheng Song ${ }^{2,4 \dagger}$, Yiran Zhang ${ }^{1,2,4}$, Weijian Liu ${ }^{3^{*}}$, Lian Zhu ${ }^{2,4^{*}}$ and Yingze Zhang ${ }^{1,2,4^{*}}$

\begin{abstract}
Background: Several studies have demonstrated a relationship between the posterior tibial slope (PTS) and meniscal tears in adults. However, little is known about the association between the PTS of the adolescents and medial meniscal tears (MMT). The purpose of this study was to evaluate the association between the PTS and MMT in adolescents, and to determine the optimal cut-off values of PTS for discriminating between the MMT and the control groups.

Methods: Between January 2018 and January 2020, a retrospective case-control study was performed. In this study, isolated MMT adolescent patients with no ligamentous injuries were matched by age and sex to a control group of radiologically normal images. The PTS was defined as the angle between the perpendicular line to proximal tibial cortex (PTC) and the tangent line along the tibial plateau. Then, both the medial posterior tibial slope (MPTS) and lateral posterior tibial slope (LPTS) were measured by plain radiographs on the lateral views. In addition, the optimal cut-off values of PTS were determined by the receiver operating characteristic (ROC) curve analysis.

Results: A total of seventy-two patients who met the inclusion criteria were enrolled in the final analysis (36 patients with isolated MMT, 36 controls). The MPTS was greater in the knees with isolated MMT $\left(10.7^{\circ} \pm 2.1^{\circ}\right)$ than that of the control group $\left(8.8^{\circ} \pm 1.7^{\circ}\right)$, showing significant difference $(P<0.001)$. However, there was no significant difference regarding the LPTS between the isolated MMT and controls (11.5 \pm 3.4 vs $10.9 \pm 2.6, p>0.05)$. In the ROC curve analysis, the calculated cutoff value of the MPTS discriminating between the groups was $10.3^{\circ}$, with a sensitivity of $73.3 \%$ and specificity of $78.9 \%$.
\end{abstract}

Conclusions: This study demonstrated that steep MPTS is associated with MMT, and MPTS $\geq 10.3^{\circ}$ was identified to be a risk factor for MMT in adolescents.

\footnotetext{
*Correspondence: xh_liuweijian@hust.edu.cn; zhulian050051@163.com;

zyz050051@163.com

${ }^{\dagger}$ Xiangtian Deng, Hongzhi Hu and Qingcheng Song contributed equally to this work

${ }^{3}$ Department of Orthopedics, Union Hospital of Tongji Medical College of Huazhong University of Science and Technology, Wuhan 430022, People's Republic of China

${ }^{4} \mathrm{NHC}$ Key Laboratory of Intelligent Orthopaedic Equipment, Third Hospital of Hebei Medical University, Shijiazhuang 050051, Hebei, People's Republic of China
}

Full list of author information is available at the end of the article permits use, sharing, adaptation, distribution and reproduction in any medium or format, as long as you give appropriate credit to the original author(s) and the source, provide a link to the Creative Commons licence, and indicate if changes were made. The images or other third party material in this article are included in the article's Creative Commons licence, unless indicated otherwise in a credit line to the material. If material is not included in the article's Creative Commons licence and your intended use is not permitted by statutory regulation or exceeds the permitted use, you will need to obtain permission directly from the copyright holder. To view a copy of this licence, visit http://creativecommons.org/licenses/by/4.0/. The Creative Commons Public Domain Dedication waiver (http://creativeco mmons.org/publicdomain/zero/1.0/) applies to the data made available in this article, unless otherwise stated in a credit line to the data. 
Keywords: Adolescent, Medial meniscal tears, Posterior tibial slope, Case-control study

\section{Background}

Meniscal tears, occurred in adolescent patients, are common injuries concomitant with anterior cruciate ligament (ACL) ruptures during athletic activity. If left untread, meniscal tears are thought to carry potential sequelae, resulting in abnormal stress distribution within the tibiofemoral joint; therefore, accelerating the deterioration of the articular cartilage and early post-traumatic osteoarthritis $[1,2]$. Overall, risk factors for meniscal tears are considered to be extrinsic and intrinsic. Extrinsic risk factors for meniscal tears is multifactorial, including neuromuscular forces, injury mechanisms, delay of surgery, untreated ligamentous injuries, and landing biomechanics during physical activity [3-5], while the intrinsic risk factors, including age, sex, lower limb alignment, high body mass index (BMI), and geometry of the tibial plateau [6-8]. Identification of these intrinsic anatomical factors, such as posterior tibial slope (PTS), may help orthopedic surgeons better recognize risk factors of meniscal tears in susceptible individuals.

In recent years, several studies have been conducted on the risk factors between the PTS and meniscal injuries in ACL-deficient knees [6, 8-12]. The bony morphology of the proximal tibia, especially a steep tibial slope, is known to have a negative effect on the biomechanics and kinematics of the knee [13, 14]. Furthermore, increased PTS is associated with the magnitude of the shear force in tibial platform and influences the biomechanics of the tibiofemoral joint, which may increase the risk of meniscal injuries in adults [10]. Actually, medial meniscal tears (MMT) are more frequently occurred due to the role in transmitting shear force with compressive force. Furthermore, medial meniscus also plays an important role in maintenance of knee stability [15-17]. Until now, however, potential anatomical risk factors for MMT in adolescents remain unclear. As is well known, meniscus is a critical regulator within the tibiofemoral joint with various functions, such as shock absorption, load diffusing, and enhancement of stability [18]. In addition, the medial meniscus is an important component that distributes compressive loading force across the knee and is subjected to anteroposterior shear forces. Walker et al. [19] reported that the medial meniscus delivers the higher percentage of shear force compared to lateral meniscus. Using knee radiographs and magnetic resonance imaging (MRI), Markl et al. [12] showed a statistically significant association between a steep PTS and MMT in adults, regardless of sex. Nevertheless, limitations of these studies are they focused on the relationship between the PTS and meniscal tears in adults. In clinical practice, it is also important for orthopedic surgeons to investigate the association between the PTS and MMT in adolescents.

Therefore, it is necessary to suppose that the morphology of the tibial may act as an important role in MMT because increased PTS would lead to higher shear force in the medial meniscus during compressive loading. To the best of our knowledge, however, there is a paucity of literatures regarding the relationship between the PTS and MMT in adolescents, despite their potential clinical importance. The purpose of this study was (a) to investigate the association between the PTS and MMT, and (b) to determine the optimal cut-off values of PTS for discriminating between the MMT and the control groups. It was hypothesized that an increased PTS may serve as a potential risk factor for MMT in this population.

\section{Methods \\ Study design}

This retrospective case-control study was performed at the Third Hospital of Hebei Medical University and approved by the local institutional ethics committee. All included patients provided written informed consent and this study complied with the principles of the Declaration of Helsinki.

Data from January 2018 to January 2020, patients who visited the hospital with knee discomfort and underwent knee radiographs and magnetic resonance imaging (MRI) scans were retrospectively reviewed, and they were divided into the two groups: isolated MMT group and control group, respectively. Inclusion criteria for isolated MMT group were as follows: a) patients younger than 16 years; b) presence of isolated MMT as visualized on MRI scan and reconfirmed with arthroscopy intraoperatively; c) good-quality of knee radiographs (with both femoral condyles completely overlapped in lateral views at approximately $30^{\circ}$ of knee flexion) and MRI scans. Patients who met the following criteria were excluded: a) concomitant with other injuries, such as ligamentous injuries, severe cartilage defects, and lateral meniscal tears; b) patients with previous trauma or knee surgery affecting the bony anatomy of the knee joint. Moreover, the control group was defined as the knee MRI scans with normal radiological images during the same period. Patients were excluded from the control group if they had any pathological images, knee malformation or malalignment, and an unavailable knee radiographs or MRI. In this case-control study, the isolated MMT cases were matched by age and sex to a group of radiologically 
normal controls. Figure 1 shows the flow diagram of patient enrollment in the study.

\section{Radiological assessments}

All patients included in the study underwent knee radiographs and MRI examination. There have been many techniques measuring PTS using different reference line in knee radiographs, and our measurement technique was based on the foundation of a previously validated method described by Hohmann et al. [20]. Two experi-

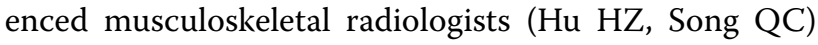
who blinded to patients' data independently assessed radiographic parameters twice using the measurement tools available in the picture archiving and communication system (PACS; Science \& Technology General Company of the Third Hospital of Hebei Medical University, shijiazhuang, China) in our institution at an interval of 2 weeks to assess the intra- and inter-observer reliability. On lateral radiographic view both the medial/lateral posterior tibial slope (MPTS/LPTS) was defined as the angle between the perpendicular line to posterior cortical line (PTC) and the tangent line connecting the most superior points at the anterior and posterior edges of the respective tibial plateau, as shown in Figs. 2 and 3. The PTC was determined by two cortical points: the points located at 5- and 15- cm distal to the knee joint line [21], respectively. Once connecting these two points, the longitudinal axis of the PTC can be represented.

\section{Statistical analysis}

SPSS software (version 24.0, IBM Corp., USA) was performed for statistical analysis. Continuous variables were tested for normality distribution by KolmogorovSmirnov test and then presented as mean \pm standard deviation. After data normality was established, parametric variables were compared using Student's $t$-test. The sample size was calculated according to a priori power analysis, and a sample size of 32 per group was considered as the minimum number for alpha of 0.05 and a power of 0.8. Receiver operating characteristic (ROC) curve analysis was performed to identify the optimal cut-off values of PTS. Also, the intra-observer and interobserver reliabilities of PTS measurements were analyzed

Patients younger than 16 years who visited the hospital between January 2018 and January 2020 with knee discomfort and underwent knee radiographs and MRI scans were retrospectively reviewed

$$
(\mathrm{n}=578)
$$

\begin{tabular}{|c|c|}
\hline $\begin{array}{l}\text { Meniscal tears with arthroscopically confirmed } \\
\text { Exclusion: }(\mathrm{n}=58) \\
\text { - Concomitant with other injuries, including } \\
\text { ligamentous injuries/cartilage injuries } \\
\text { /lateral meniscal tears }(\mathrm{n}=17) \\
\text { - Previous trauma or knee surgery }(\mathrm{n}=5)\end{array}$ & $\begin{array}{l}\quad \text { Control group }(\mathrm{n}=520) \\
\text { Exclusion: } \\
\text { - Patients with pathological images, } \\
\text { visualized using MRI }(\mathrm{n}=86) \\
\text { - An unavailable knee radiograph and MRI } \\
\text { scan }(\mathrm{n}=49) \\
\text { - Knee malformation or malalignments }(\mathrm{n}=13)\end{array}$ \\
\hline Isolated medial meniscal tears $(n=36)$ & Control group $(n=372)$ \\
\hline \multicolumn{2}{|c|}{ After matching of age and gender } \\
\hline Isolated medial meniscal tears $(\mathrm{n}=36)$ & Control group $(\mathrm{n}=36)$ \\
\hline
\end{tabular}

Fig. 1 Flowchart illustrating the enrollment of patients for this study 


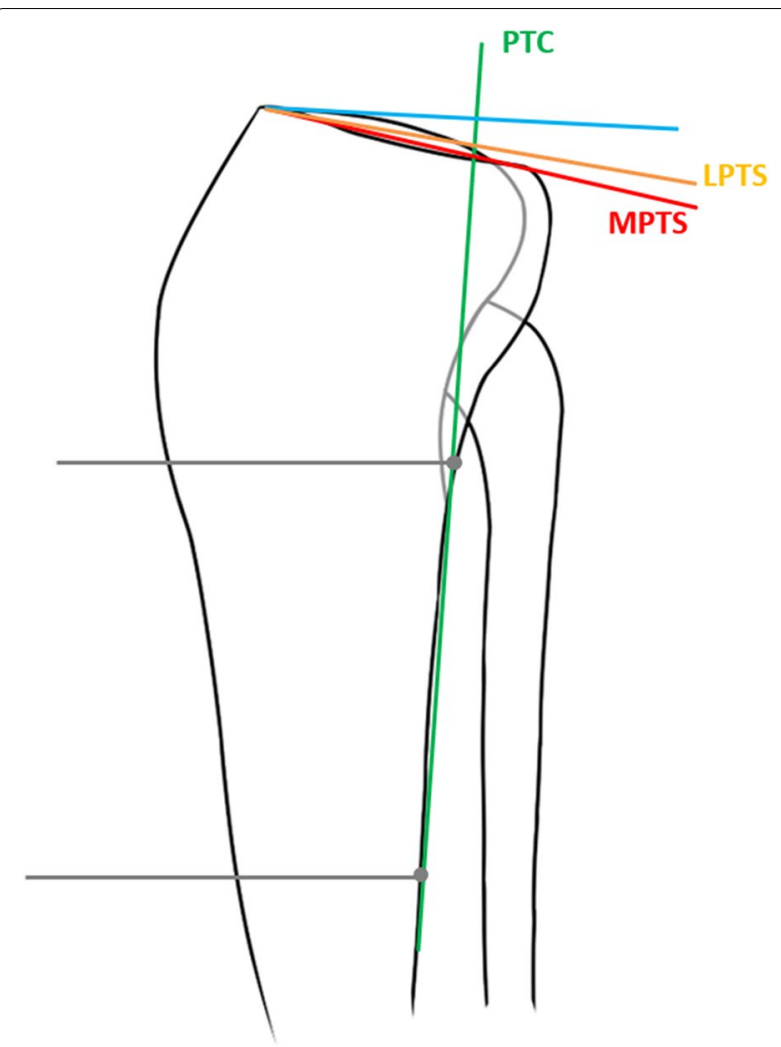

Fig. 2 Schematic illustration showing the descried measurement technique for calculating medial and lateral tibial posterior tibial slope on lateral views. MPTS, medial posterior tibial slope; LPTS, lateral posterior tibial slope. PTC, proximal tibial cortex

by intraclass correlation coefficients (ICCs). For all tests, statistical significance level was set at $p<0.05$.

\section{Results}

Twenty-two patients in the isolated MMT group were excluded due to ligamentous injuries, cartilage defects, lateral meniscal tears, and previous trauma or knee surgery. A total of seventy-two patients who met the inclusion and exclusion criteria were enrolled in the final analysis (36 with isolated MMT, and 36 controls). All cases in the MMT group sustained sprots-related meniscal tears. Each group consisted 21 males and 15 females. As shown in Table 1, the ICCs for the MPTS was 0.912 (95\% CI, 0.875-0.954) for the intra-observer reliability and 0.894 (95\% CI, 0.834-0.937) for the inter-observer reliability, and the ICCs for the LPTS was 0.878 (95\% CI, $0.847-0.921$ ) for the intra-observer reliability and 0.851 (95\% CI, 0.804-0.913) for the inter-observer reliability, indicating an excellent inter- and intra-reliabilities.

The patients' demographic characteristics and PTS measurements of the two groups were shown in Table 2.
No significant difference was observed between the two groups in terms of age $(p=0.566)$ and genders $(p=1.000)$. The MPTS was higher in the knees with isolated MMT than that of the controls $\left(10.7^{\circ} \pm 2.1^{\circ}\right.$ vs $8.8^{\circ} \pm 1.7^{\circ}$, $p<0.001)$. However, there was no significant difference regarding the LPTS between the isolated MMT and controls $(11.5 \pm 3.4$ vs $10.9 \pm 2.6, p=0.403)$. To clarify the exact tear location of the MM, the meniscus was divided into three parts: the anterior horn $(\mathrm{AH})$, the body, and the posterior horn $(\mathrm{PH})$. In our study, the most common tear location of the MM was the posterior horn, followed by the tears involving the body. In addition, tears of the anterior parts of the menisci were seen least frequently. The distribution of meniscal tears in different anatomical location was shown in Fig. 4. According to the ROC analysis, the calculated optimal cut off value of MPTS with an increased risk for isolated MMT was $10.3^{\circ}$, with a sensitivity of $73.3 \%$ and specificity of $78.9 \%$ (Fig. 5).

\section{Discussion}

The most important finding of the present study was that increased MPTS is associated with an increased risk for isolated MMT, whereas the LPTS appears to have less of an influence. These findings are similar with existing studies involving the adult population [22]. In addition, our results showed that the cut-off values of MPTS discrimination between the groups in adolescent patients was $10.3^{\circ}$, with a sensitivity of $73.3 \%$ and a specificity of $78.9 \%$. This association may be originated from the biomechanical change and higher shear force in tibial plateau under the condition of a steep tibial slope. It has been reported that the tibial slope was an anamical factor which have a great influence on the kinematics of tibiofemoral joint [23]. A large number of biomechanical studies have also shown a liner relationship between steep tibial slope and anterior translation of the tibia during weightbearing activities, which subsequently increased shear forces on ACL and meniscus [14, 24, 25]. Therefore, a steep tibial slope, leading to an increased anterior tibial translation, may predispose adolescent patients to MMT.

Actually, multiple literatures have shown that an association between increased PTS and meniscal tears in adults $[11,22,26]$. Kolbe et al. [11] identified that increased LPTS is a potential risk factor for posterolateral meniscal root tears in ACL-deficient knees. Moreover, Moon et al. [22] demonstrated that patients with medial meniscal posterior horn tears had significantly greater PTS than controls. Alici et al. [26] utilized both knee radiographs and MRI scans to measure PTS values in 212 meniscal tears patients, and they reported that the lateral meniscal tears group had increased LPTS when compared to the 


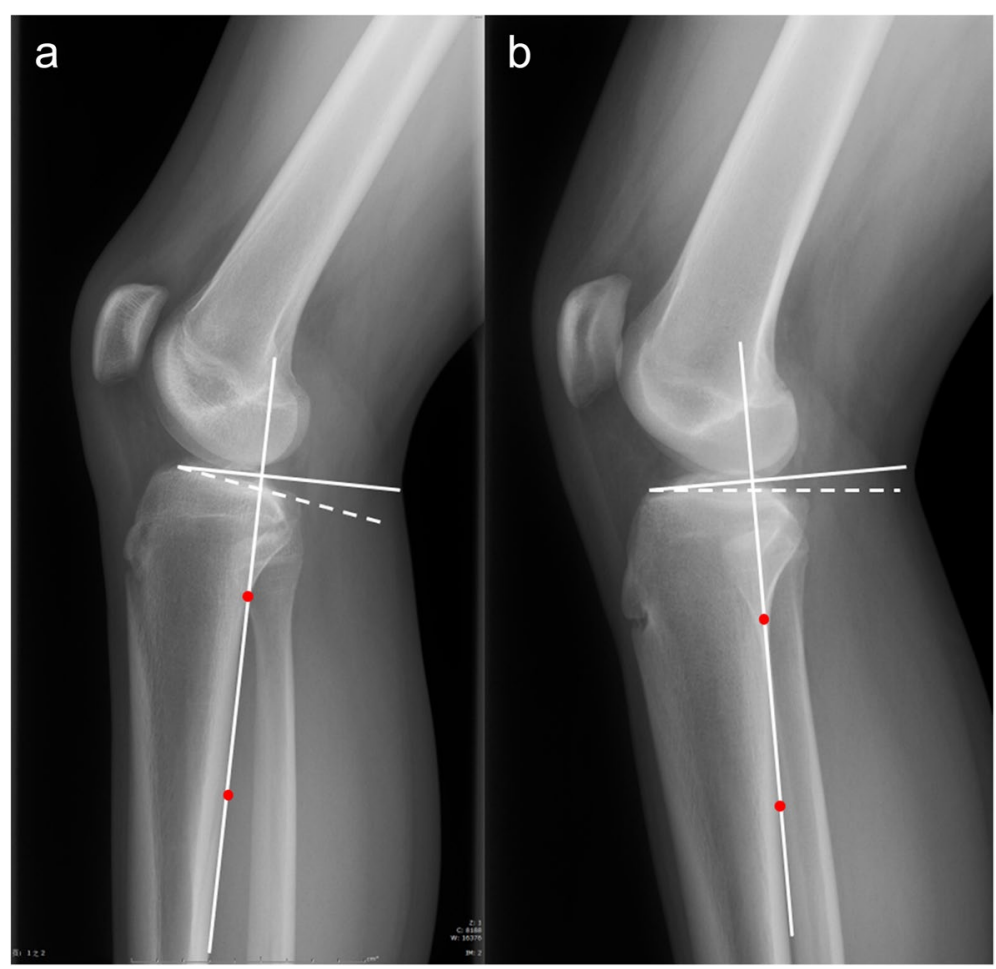

Fig. 3 The PTS is defined as the angle between the dash line drawn along the anterior and posterior edges of the tibial plateau and the line perpendicular to the PTC. An example of the MPTS in (a) the MMT group ("actual degree") and (b) control group ("actual degree"). MMT, medial meniscal tear; PTC, posterior tibial line; MPTS, medial posterior tibial slope

Table 1 Intra-observer and inter-observer reliability among PTS measurements performed ${ }^{\mathrm{a}}$

\begin{tabular}{lll}
\hline Variables & $\begin{array}{l}\text { Intra-observer reliability } \\
\text { ICC }(\mathbf{9 5 \%} \mathbf{C l})\end{array}$ & $\begin{array}{l}\text { Inter-observer reliability } \\
\text { ICC }(\mathbf{9 5 \%} \mathbf{C l})\end{array}$ \\
\hline MPTS & $0.912(0.875-0.954)$ & $0.894(0.834-0.937)$ \\
LPTS & $0.878(0.847-0.921)$ & $0.851(0.804-0.913)$ \\
\hline
\end{tabular}

ICC intraclass correlation coefficient, MPTS medial posterior tibial slope, LPTS lateral posterior tibial slope

${ }^{\text {a }}$ Values are presented as intraclass correlation $(95 \% \mathrm{Cl})$

Table 2 Patients' demographic characteristics and measurements between MMT cases and healthy controls

\begin{tabular}{llll}
\hline & MMT group & Control group & P value \\
\hline Patients & 36 & 36 & \\
Age (years) & $15.0 \pm 1.6$ & $14.8 \pm 1.2$ & 0.566 \\
Male/female & $21 / 15$ & $21 / 15$ & 1.000 \\
MPTS $\left(^{\circ}\right)$ & $10.7 \pm 2.1$ & $8.8 \pm 1.7$ & $<0.001^{*}$ \\
LPTS $\left(^{\circ}\right)$ & $11.5 \pm 3.4$ & $10.9 \pm 2.6$ & 0.403 \\
\hline
\end{tabular}

Control group, patients without any pathologic findings on MRI

Continuous variables are presented as mean \pm standard deviation

MMT medial meniscal tears, MPTS medial posterior tibial slope, LPTS lateral posterior tibial slope

${ }^{*}$ Statistically significant $(P<0.05)$

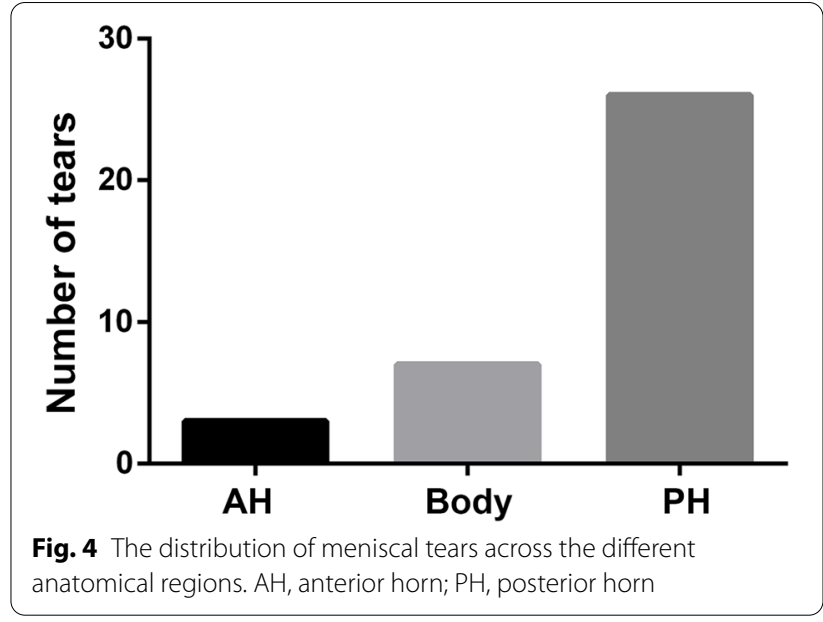

control group, whereas there is no association between the increased PTS values and MMT. These abovementioned published results provided some evidences that MMT are associated with increased tibial slope.

Despite the growing interests in PTS and its relationship with meniscal tears in adults [22, 27], this intrinsic anatomic factor remains unclear in adolescent patients. An increased demand to attain athletic activities and 


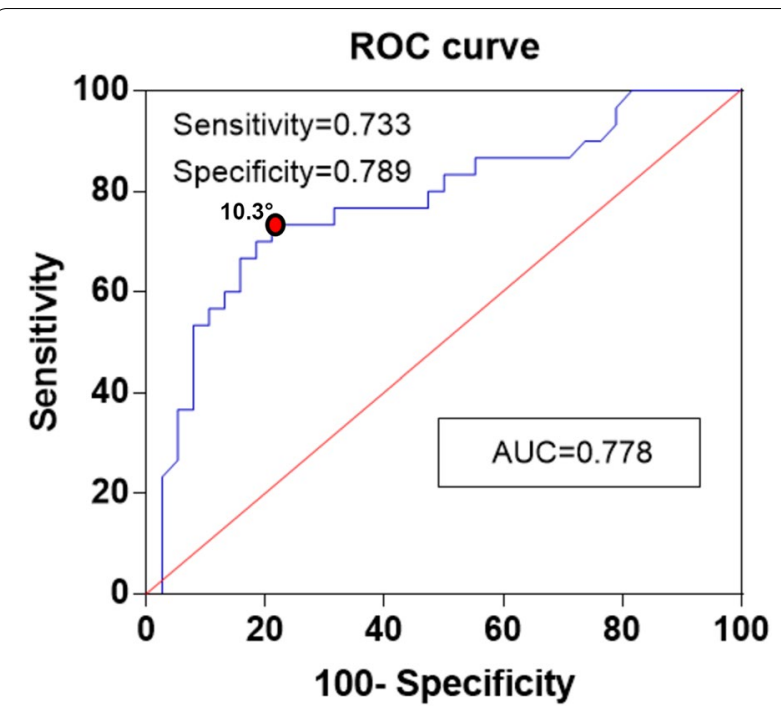

Fig. 5 Receiver operating characteristic curve analysis for MPTS measurement. Reference line (diagonal): $A \cup C=0.778$. AUC, area under the curve; MPTS, medial posterior tibial slope

peak performance in adolescent patients may result in higher incidence of meniscal tears, especially in patients with increased tibial slope. The current study showed significant difference in MPTS values between the isolated MMT group and control group. Also, these findings were consistent with the results of Moon et al. [22], who demonstrated significant differences in MPTS between medial meniscal posterior horn tears patients and matched controls. Similarly, we assumed that this phenomenon may be partially explained by the function of medial meniscus, sustaining higher shear force and compressive loading forces in the presence of physiologic activities [28]. Therefore, anterior translation of the tibial that originate from a steep tibial slope may have a greater influence on medial meniscus. Of note, the main clinical significance of this study may have profound effects in the establishment of identification risk factors of MMT in a plain radiograph without using MRI.

The optimal cut-off value of MPTS was determined with the ROC curve analysis, which showed that the MPTS greater than $10.3^{\circ}$ is associated with an increased risk for isolated MMT in adolescent patients. Recently, Moon et al. [22] identified that increased PTS $\left(\geq 6.6^{\circ}\right)$ was considered as an anatomical predictor for medial meniscal posterior horn tears with sensitivity of $55.3 \%$ and specificity of $75.0 \%$. The reason for this discrepancy may be explained by the difference of patient selection. Dare et al. [29] revealed that PTS was tend to decrease or flatten with age, which may explain that the cut-off PTS values of the study was significantly higher than previously reported in adults. Another reason for this discrepancy may be attributed to the racial differences. Taken together, we considered that the adverse effect by increased tibial slope on MMT should not be overlooked in adolescents.

Several limitations should be noted in the present study. First, it was a retrospective study with the usual limitations and bias inherent to single center. To minimize bias, all observers who measured MPTS and LPTS on radiographs were blinded to the arthroscopic findings of meniscal status. Second, the number of patients enrolled in our study was relatively small and the location of meniscal tears were not evaluated in this study. However, the results of the presents study are meaningful in clinical significance, as the cut-off values of MPTS found in our study may be helpful for surgeons to consider intrinsic anatomical risk factors for MMT, especially with increasing number of meniscal tears in adolescents. Despite these limitations, there were important strengths associated with this study. To our knowledge, this is the first study to investigate the relationship between MPTS and MMT in adolescent patients. Also, these characteristics should be assessed by orthopedic surgeons using plain radiographs when suspect MMT in the adolescent patients.

\section{Conclusions}

In conclusion, this study demonstrated that increased MPTS is associated with MMT, and MPTS $\geq 10.3^{\circ}$ was identified to be a risk factor for MMT in adolescents. This finding places an emphasis on the identification of risk factors for MMT, that is, if we recognize the steep MPTS in a plain radiograph, we can strongly suspect risk of MMT with using MRI.

\section{Abbreviations \\ PTS: Posterior tibial slope; MPTS: Medial posterior tibial slope; LPTS: Lateral posterior tibial slope; MMT: Medial meniscal tears; MRI: Magnetic resonance imaging; ROC: Receiver operating characteristic curve; ACL: Anterior cruciate ligament; PTC: Proximal cortical line; PACS: Picture archiving and communica- tion system; ICCs: Intraclass correlation coefficients.}

\section{Acknowledgements}

Not applicable.

\section{Authors' contributions}

YZZ designed the study; XTD, HZH, and QCS searched relevant studies; YRZ, WJL and LZ analyzed and interpreted the data; XTD wrote the manuscript; and $L Z$ and $Y Z Z$ approved the final version of the manuscript. The authors read and approved the final manuscript.

\section{Funding}

None.

\section{Availability of data and materials}

All the data and material involving this article will be available upon request by send an e-mail to the first author. 


\section{Declarations}

Ethics approval and consent to participate

This study was approved by the ethics committee of the Third Hospital of Hebei Medical University. Informed consent was obtained from all individual participants included in the study.

\section{Consent for publication}

Consent to publish was obtained from each patient.

\section{Competing interests}

All the authors declare that they have no conflict of interest with any organization.

\section{Author details}

'School of Medicine, Nankai University, Tianjin 300071, People's Republic of China. ${ }^{2}$ Department of Orthopaedic Surgery of Hebei Province, Third Hospital of Hebei Medical University, 139 Ziqiang Road, Shijiazhuang 050051, Hebei, People's Republic of China. ${ }^{3}$ Department of Orthopedics, Union Hospital of Tongji Medical College of Huazhong University of Science and Technology, Wuhan 430022, People's Republic of China. ${ }^{4}$ NHC Key Laboratory of Intelligent Orthopaedic Equipment, Third Hospital of Hebei Medical University, Shijiazhuang 050051, Hebei, People's Republic of China.

Received: 8 July 2021 Accepted: 7 October 2021

Published online: 25 October 2021

\section{References}

1. Thorlund JB, Juhl CB, Ingelsrud LH, Skou ST. Risk factors, diagnosis and non-surgical treatment for meniscal tears: evidence and recommendations: a statement paper commissioned by the Danish Society of Sports Physical Therapy (DSSF). Br J Sports Med. 2018;52(9):557-65.

2. Beynnon BD, Hall JS, Sturnick DR, Desarno MJ, Gardner-Morse M, Tourville TW, et al. Increased slope of the lateral tibial plateau subchondral bone is associated with greater risk of noncontact ACL injury in females but not in males: a prospective cohort study with a nested, matched case-control analysis. Am J Sports Med. 2014;42(5):1039-48.

3. Kay J, Memon M, Shah A, Yen YM, Samuelsson K, Peterson D, et al. Earlier anterior cruciate ligament reconstruction is associated with a decreased risk of medial meniscal and articular cartilage damage in children and adolescents: a systematic review and meta-analysis. Knee Surg Sports Traumatol Arthrosc. 2018;26(12):3738-53.

4. Ekas GR, Ardern C, Grindem H, Engebretsen L. New meniscal tears after ACL injury: what is the risk? A systematic review protocol. Br J Sports Med. 2018;52(6):386.

5. Guenther ZD, Swami V, Dhillon SS, Jaremko JL. Meniscal injury after adolescent anterior cruciate ligament injury: how long are patients at risk? Clin Orthop Relat Res. 2014:472(3):990-7.

6. Raad M, Thevenin LC, Berard E, Laumonerie P, Sales DGJ, Accadbled F. Delayed reconstruction and high $\mathrm{BMI} z$ score increase the risk of meniscal tear in paediatric and adolescent anterior cruciate ligament injury. Knee Surg Sports Traumatol Arthrosc. 2019;27(3):905-11.

7. Mansori AE, Lording T, Schneider A, Dumas R, Servien E, Lustig S. Incidence and patterns of meniscal tears accompanying the anterior cruciate ligament injury: possible local and generalized risk factors. Int Orthop. 2018;42(9):2113-21.

8. Song GY, Liu X, Zhang H, Wang QQ, Zhang J, Li Y, et al. Increased medial meniscal slope is associated with greater risk of ramp lesion in noncontact anterior cruciate ligament injury. Am J Sports Med. 2016;44(8):2039-46.

9. Kim SH, Seo HJ, Seo DW, Kim KI, Lee SH. Analysis of risk factors for ramp lesions associated with anterior cruciate ligament injury. Am J Sports Med. 2020;48(7):1673-81.

10. Lee JJ, Choi YJ, Shin KY, Choi CH. Medial meniscal tears in anterior cruciate ligament-deficient knees: effects of posterior tibial slope on medial meniscal tear. Knee Surg Relat Res. 2011;23(4):227-30.

11. Kolbe R, Schmidt-Hebbel A, Forkel P, Pogorzelski J, Imhoff AB, Feucht MJ. Steep lateral tibial slope and lateral-to-medial slope asymmetry are risk factors for concomitant posterolateral meniscus root tears in anterior cruciate ligament injuries. Knee Surg Sports Traumatol Arthrosc. 2019;27(8):2585-91.

12. Markl I, Zantop T, Zeman F, Seitz J, Angele P. The effect of tibial slope in acute ACL-insufficient patients on concurrent meniscal tears. Arch Orthop Trauma Surg. 2015;135(8):1141-9.

13. Lipps DB, Oh YK, Ashton-Miller JA, Wojtys EM. Morphologic characteristics help explain the gender difference in peak anterior cruciate ligament strain during a simulated pivot landing. Am J Sports Med. 2012:40(1):32-40

14. Dejour $\mathrm{H}$, Bonnin M. Tibial translation after anterior cruciate ligament rupture. Two radiological tests compared. J Bone Joint Surg Br. 1994;76(5):745-9.

15. Allen CR, Wong EK, Livesay GA, Sakane M, Fu FH, Woo SL. Importance of the medial meniscus in the anterior cruciate ligament-deficient knee. J Orthop Res. 2000;18(1):109-15.

16. Elmansori A, Lording T, Dumas R, Elmaji K, Neyret P, Lustig S. Proximal tibial bony and meniscal slopes are higher in $\mathrm{ACL}$ injured subjects than controls: a comparative MRI study. Knee Surg Sports Traumatol Arthrosc. 2017:25(5):1598-605

17. Grassi A, Di Paolo S, Lucidi GA, Macchiarola L, Raggi F, Zaffagnini S. The contribution of partial Meniscectomy to preoperative laxity and laxity after anatomic single-bundle anterior cruciate ligament reconstruction: in vivo kinematics with navigation. Am J Sports Med. 2019;47(13):3203-11.

18. Hutchinson ID, Moran CJ, Potter HG, Warren RF, Rodeo SA. Restoration of the meniscus: form and function. Am J Sports Med. 2014;42(4):987-98.

19. Walker PS, Arno S, Bell C, Salvadore G, Borukhov I, Oh C. Function of the medial meniscus in force transmission and stability. J Biomech. 2015;48(8):1383-8.

20. Hohmann E, Bryant A, Reaburn P, Tetsworth K. Is there a correlation between posterior tibial slope and non-contact anterior cruciate ligament injuries? Knee Surg Sports Traumatol Arthrosc. 2011;19(Suppl 1):S109-14.

21. Utzschneider S, Goettinger M, Weber P, Horng A, Glaser C, Jansson V, et al. Development and validation of a new method for the radiologic measurement of the tibial slope. Knee Surg Sports Traumatol Arthrosc. 2011:19(10):1643-8

22. Moon HS, Choi CH, Jung M, Lee DY, Eum KS, Kim SH. Medial meniscal posterior horn tears are associated with increased posterior Tibial slope: a case-control study. Am J Sports Med. 2020;48(7):1702-10.

23. Hashemi J, Chandrashekar N, Gill B, Beynnon BD, Slauterbeck JR, Schutt RJ, et al. The geometry of the tibial plateau and its influence on the biomechanics of the tibiofemoral joint. J Bone Joint Surg Am. 2008;90(12):2724-34

24. Giffin JR, Vogrin TM, Zantop T, Woo SL, Harner CD. Effects of increasing tibial slope on the biomechanics of the knee. Am J Sports Med. 2004;32(2):376-82.

25. Shelburne KB, Kim HJ, Sterett WI, Pandy MG. Effect of posterior tibial slope on knee biomechanics during functional activity. J Orthop Res. 2011;29(2):223-31.

26. Alici T, Esenyel CZ, Esenyel M, Imren Y, Ayanoglu S, Cubuk R. Relationship between meniscal tears and tibial slope on the tibial plateau. Eurasian J Med. 2011;43(3):146-51.

27. Hiranaka T, Furumatsu T, Okazaki Y, Yamawaki T, Okazaki Y, Kodama Y, et al. Steep medial tibial slope and prolonged delay to surgery are associated with bilateral medial meniscus posterior root tear. Knee Surg Sports Traumatol Arthrosc. 2021;29(4):1052-7.

28. Hudek R, Fuchs B, Regenfelder F, Koch PP. Is noncontact ACL injury associated with the posterior tibial and meniscal slope? Clin Orthop Relat Res. 2011;469(8):2377-84.

29. Dare DM, Fabricant PD, McCarthy MM, Rebolledo BJ, Green DW, Cordasco FA, et al. Increased lateral Tibial slope is a risk factor for pediatric anterior cruciate ligament injury: an MRI-based case-control study of 152 patients. Am J Sports Med. 2015;43(7):1632-9.

\section{Publisher's Note}

Springer Nature remains neutral with regard to jurisdictional claims in published maps and institutional affiliations. 